\title{
Research on Visual Communication and Design Performance of Web Page
}

\author{
Li guiping \\ Institute of Art and Design, Yancheng Normal College, Yancheng, Jiangsu, 224000, China
}

Keywords: web design; visual elements; visual communication

\begin{abstract}
In recent years, with the development of the network, the web page as a carrier of the network, has conveyed the various information in a quick and convenient form to the audience. In this case, people gradually produced a demand for web aesthetics. People's pursuit of beauty is deepening, the same as the web design. From the web design of the visual elements of the function and its aesthetic performance point of view, it is necessary to consider how to more effectively convey information, to leave a good impression to users, to improve the site visits, and promote the good operation of the site.
\end{abstract}

\section{The Functional Requirements of Page on the Visual Elements}

Network as a media form is a combination of graphics, text, sound, animation and other information, to provide people with a convenient information exchange platform. The expression of visual information in webpage becomes an important part of network information communication. In recent years, the visual performance of web pages has evolved from the simple map and the form of the arrangement, to the integrated use of multiple visual elements. The new web design under the new media makes the effect of visual communication a new look. On the current web design status, the development of web design is still in a state of unsatisfactory, such as low bandwidth, web design staff level is limited, the computer programming technology is not high. The web design is still in a text not really can attract the interests of Internet users, such as audio, video, pictures, animation and other forms have not been fully used, lack of artistic design of web design, its functionality has not been a good expression.

\section{The Issues of Visual Design of Web Design}

In the current web design, there is some research and practical experience in the recognition and convenience of visual information communication, but it is rare to be able to successfully absorb and apply the results of web design. Web page visual expression is more single, the page link cumbersome, these factors seriously hinder the effective dissemination of network information, so that information cannot be effectively communicated to the user, and is not conducive to communicate with each other between users.The visual effects and the page layout are clearly different.The impact of information design structure and content, visual effects can rely on the nature of the site, customer interest, web designer personality was rich and varied. "[1] for different types of sites, designers should clearly take what kind of approach to make the performance, in order to achieve the appropriate purpose of communication.

Web design is no longer a purely medium, it has become an art form. At present, China's web design to be developed, relatively speaking,the development of foreign web design in theory and practice has been relatively sound, visual performance more harmonious unity. Therefore, the analysis of visual information in web design to improve the click-through rate and the aesthetic awareness of the people has a very important significance. Web design is based on the purpose of the site, the structure, graphic information, color and other visual elements organically unified in the page, and the overall style of the page design, the use of reasonable layout elements accurate, systematic and effective communication of information. Web page layout design, is limited in the 
screen space will be audio-visual multimedia elements of the organic arrangement of the combination of rational thinking personalized performance, is a kind of personal style and artistic characteristics of audio-visual communication. It conveys the information at the same time, also produces sensory beauty and spiritual enjoyment. In order to make the web page to obtain the largest visual communication function, make it truly become a readable and innovative Internet carrier, web design must adapt to the visual and physiological characteristics of people's visual processes to determine the relationship between the various visual elements and order. Visual function and the form of the United States, will directly affect the information to convey the accuracy and effectiveness. Visual elements include graphics, text and color, due to the virtual space characteristics of the page, in the web design visual elements evolved richer, such as dynamic information, audio and video, etc.

\section{The Visual Design of Web Design}

To observe the visual laws of web design, can improve the visual communication of web pages. The visual principles of web design include: the unity of form and content, highlighting the theme elements. First, the unity of form and content.Requirements of the form of performance must meet the function of the visual process can naturally arrive at the information in an orderly manner, in the overall design of the web page requires the same existence. Second, highlighting the theme elements. The use of the focus of the visual process to highlight the theme, so that the theme elements will make the viewer's sight has a beginning and end. With a clear, beautiful, the overall unity of the visual effects presented to the viewer, so that in the browsing process they can happily receive information.

People in reading, there is a natural flow habits of the visual, but this kind of visual habits can be affected by visual elements. The formation of the visual process is determined by the visual characteristics of human beings, limited by the physiological structure, the human eye can only produce a focus, not at the same time to stay in two or more line of sight, we can only in accordance with a certain order browse. Although the web page is a dynamic visual process, many of the rules in graphic design also apply to web design, such as balance, rhythm and so on. The square is diagonally intersecting at an intersection, which is the focal point of vision. "The attraction of the square center is greater than the attraction of the vertex of the corners." [2] In general, our vision is from left to right, from the top to down to see, so a blank page to bring us the natural visual process is from the top left to the bottom right of a curved curve. In this arc curve, the visual advantage decreases from top to bottom. In the web design, we should pay attention to the application of the grid system. The grid system from Europe can make all kinds of information elements reasonably distribute and can quickly layout, maintain a certain sense of rhythm and beauty in position, gap and size. Maintaining the unity of visual elements. Through the grid system analysis, it helps us to arrange the location and distribution of visual elements in the web page reasonably. Surrounded by white, the central location to become the focus of the visual, so that the information to be expressed more clearly and strongly. The home page according to this habit, the design of the company's important information "logo" into the upper left corner of the location, and then press the importance of placing other content. So that the overall image of the site uniform, consistent style, through repeated visual stimulation to the viewer left a deep and unified website impression.

\section{The features and aesthetic of layout in the page}

Web page layout function, mainly in order to meet people's practical needs, people interact with the page more smoothly. This requires three points: first, primary and secondary, highlight the focus. In the early stage of the web design, it should be clear what the purpose of the site, each site will have their own ultimate goal, highlight their own characteristics, clear what the site should say, how to say. Second,navigation clear and beautiful, easy to browse. Navigation in the page is very important, navigation clear, beautiful decision to browse the smooth. In the design should be as much as possible between the various pages of the page switch between easy. Third, good use of 
graphics. The use of graphics in web design is more common, these graphics guide the user to view the required information, there are abstract and figurative points, such as: site logo, abstract graphics with guide buttons, text symbols, etc., each small shape can play Guide the role of information transmission, these graphics can be summarized as a page on the point, line, surface, affecting the visual aesthetic of the page. In the web design, to carefully analyze the size of these graphic elements, location, color level, unified style, seeking common ground. Good layout design can be based on web creative, creative and carefully arranged and designed, and ultimately with excellent visual form, the formation of a beautiful style, full of good flavor visual effects, and instantly capture the viewer's eyes, giving a deep impression.

\section{Different types of website pages}

The function of the visual element and the type of the aesthetic website are qualitatively based on the service of the website. It can be divided into public service, education and learning, resource sharing, commercial operation and personal website. The function and aesthetic of different types of web design elements are different, such as the arrangement of the structure, the use of color, image and text settings. According to the theme of the site set the visual elements of different performance, the respective functions and forms in the page which together.

Commercial website. From the most basic function of the analysis is for the production of goods and transactions. Shanghai business website page, in the structure of the arrangement is more monotonous, which pays attention to product introduction and purchase order. Information content on the arrangement is tight, the use of color is relatively simple and rigid, and there are no level changes, although the entire page arrangement is order, the visual performance is very monotonous, and text content is too numerous.

Public service website. There are common sites like government agencies. This kind of website information is mostly news and publicity information, the whole page style is more formal, due to a large number of advertising, the structure is clear, the visual element is not much decoration. These pages view is simple, clear, formal with a sense of seriousness, increased the credibility of the content. But the sense of form is not rich enough, easy visual fatigue, fluttering the window to disturb the line of sight, be careful to use.

\section{The method to improve the effectiveness of web design of visual information}

First, deal with the contradiction between the effectiveness of web pages and speed. There are a number of design solutions for web page delivery speed limitations, but while using these technologies, it is necessary to be careful to make the page as simple as possible. First check the page in the HTML file structure is too complicated. Because the simple HTML file formed by the page can make the browser faster to complete a variety of data to read the work, try to use a simple table design. Guaranteed image quality while reducing image files. Second, the comprehensive and efficient use of multimedia technology. Multimedia, a variety of media communication carrier, such as flat printing, network, television, movies, mobile phones, business, etc., is a comprehensive information resources. Its basic feature is a beautiful page layout, vivid picture and picture effects, color harmony of dynamic video, flexible and convenient means of interaction. Users can not only through the text information, but also by seeing the image and hear the sound to understand the object of interest, participation or change the information presentation. Third, to increase the interaction. Interactive to fully mobilize the participation of people in the network environment from the passive development to active, so as to fully realize the individual needs of each person. In the web page information through special information navigation, so that users can get a deeper understanding and acceptance, to make faster decisions. For example, in interactive 3D advertising, consumers interact with virtual products or virtual environments to produce realistic sense of the scene, this sense of the scene can enhance consumer awareness, change the psychological and behavior of consumers, which in a single HTML environment is impossible to achieve. 


\section{References}

[1] [Korea] Cui Meishan. Ma Xiaoyang, Liu Juan translation. Designers Talk about Web Visual Effects [M]. Beijing: Electronic Industry Press, 2006.P02.

[2] [The United States] Rudolph Alnheim. TengShouyao, Zhu Jiangyuan translation. Art and Visual perception [M]. Chengdu: Sichuan people’s Publishing House. 2005.P6. 